\title{
Measurement of Capillary Length from 3D Images Acquired by Confocal Microscopy using Image Analysis and Stereology
}

\author{
L. Kubínová,* J. Janáček, *, I. Eržen, ** and X.W. Mao*** \\ * Institute of Physiology, Academy of Sciences of the Czech Republic, v.v.i., Vídeňská 1083, CZ- \\ 142 20, Prague, Czech Republic \\ ** Institute of Anatomy, Faculty of Medicine, University of Ljubljana, Korytkova 2, 1000 Ljubljana, \\ Slovenia \\ *** Loma Linda University Medical Center, 11175 Campus St., Loma Linda, CA 92354
}

Studies of the microvascular bed, i.e. an interconnected net of thin capillaries, characterized by the capillary length or length density, is of interest in many biomedical studies. 3D images acquired by a confocal microscope represent useful data for 3D reconstruction and measurements of the capillary bed, if suitable image analysis pre-processing and segmentation algorithms as well as methods for capillary measurements are applied.

We tested several methods for the length estimation on two specific examples of capillary network: 1. Capillaries in two different rat brain regions, forming a spatial net of tubules, whether denser, as it was in the case of cortex, or less dense, as in corpus callosum region. 2. Capillary bed of individual rat skeletal muscle fibers forming an anisotropic net of tubules, with axis of anisotropy going parallel to the axis of the muscle fiber. In both examples we used 3D images of capillary network acquired by a confocal microscope.

We compared three types of methods: 1. Stereological methods for the length estimation based on computer generation of isotropic uniform random virtual test probes in $3 \mathrm{D}$, either in the form of spatial grids of virtual "slicer" planes (Fig. 1a) using global spatial sampling [1,2], or sphere probes [3] (Fig. 1b). 2. Automatic method, exploiting a digital version of the Crofton relations using the Euler characteristic of planar sections of the binary image in three perpendicular principal directions [4,5]. A suitable 3D image pre-processing for compensation of inhomogeneities in image contrast, removal of image noise and enhancement of the relevant features by filtering, followed by segmentation of capillaries, was applied before using this method (Fig. 2a,b). 3. Interactive "tracer" method for the length measurement based on manual delineation of axes of capillary segments in 3D using visualization of the confocal image stack in three orthogonal sections so that each capillary segment was represented by a chain of line segments in 3D [6] (Fig. 2c). We implemented all algorithms and methods as special plug-in modules in Ellipse (ViDiTo, Slovakia) software environment.

The presented methods were compared from the point of view of their practical applicability, efficiency and precision, with respect to the arrangement of the capillary network under study. It was shown that the "slicer" and sphere probe method yielded similar results for all examined types of capillary bed and their efficiency was also comparable. The results obtained by the automatic Crofton method, interactive "tracer" and stereological methods were also in agreement, however the automatic segmentation had to be carefully adjusted. In the case of images of lower quality, e.g. too noisy, having too high background or poorly stained, automatic method is not adequate and interactive, though more time consuming methods should be applied. 
References

[1] J.O. Larsen et al., J. Microsc. 191 (1998) 238.

[2] L. Kubínová et al., J. Muscle Res. Cell Motil. 22 (2001) 217.

[3] P.R. Mouton et al., J. Microsc. 206 (2002) 54.

[4] F. Meyer, J. Microsc. 165 (1992) 5.

[5] L. Kubínová et al., in A. Diaspro, Confocal and Two-photon Microscopy, Wiley-Liss, New York, (2002) 299.

[6] Janáček et al., J. Histochem. Cytochem. 57 (2009) 437.

[7] This research was supported by the Academy of Sciences of the Czech Republic (grant AV0Z 50110509), by the Ministry of Education, Youth, and Sports of the Czech Republic (grant LC06063 and KONTAKT grants ME09010, MEB090910), and by the Grant Agency of the Czech Republic (grant No. 304/09/0733).

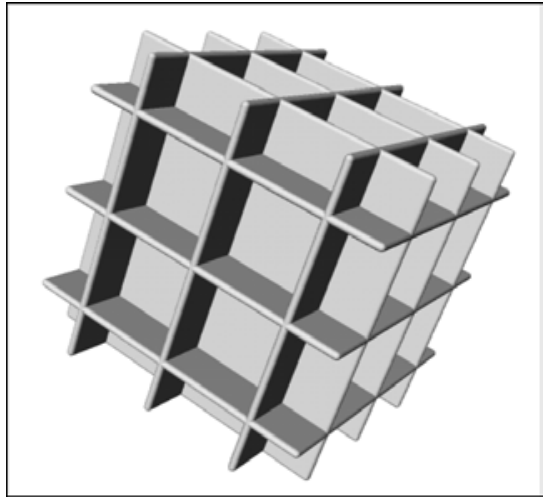

(a)

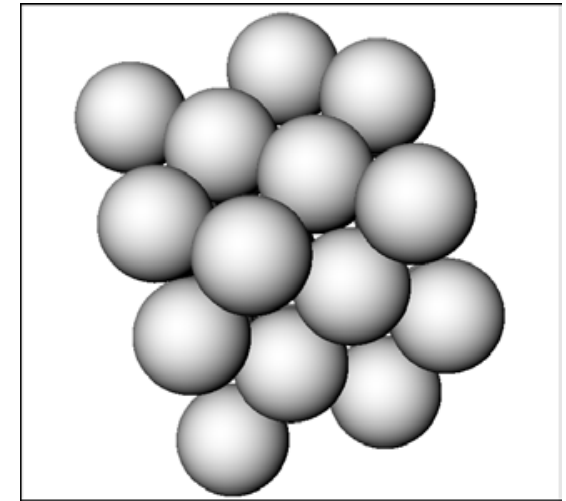

(b)

FIG. 1. Spatial grids of test probes in 3D used for length estimation. (a) Orthogonal triplet of slicer probes. (b) Spherical spatial grid.

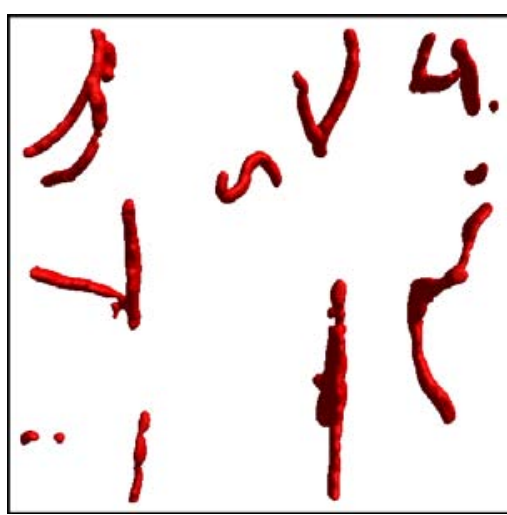

(a)

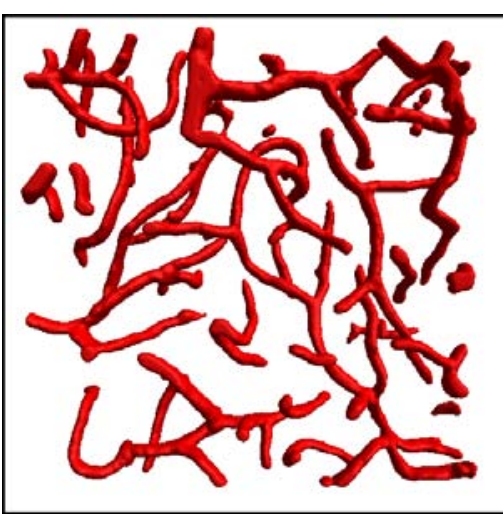

(b)

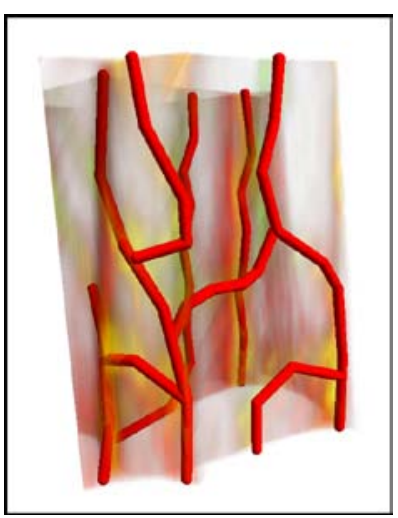

(c)

FIG. 2. Types of capillary network used for testing methods for length estimation. (a,b) Surface rendering of capillaries in the rat brain corpus callosum (a) and cortex (b) after image pre-processing by Gaussian and Lipschitz top-hat filters and segmentation by thresholding. (c) Volume rendering of a stack of confocal microscopy images of rat skeletal muscle fibre with adjacent capilaries delineated by "tracer" method. 